\title{
REVISÃo de Literatura Avaliando a fidelidade de intervenções psicossociais:
} uma revisão sistemática da literatura

\author{
Assessing fidelity in psychosocial interventions: a systematic literature review \\ Tatiana Fernandes Carpinteiro da Silva'
}

\section{RESUMO}

Objetivo: Intervenções psicossociais têm tido impacto positivo na vida das pessoas com transtorno mental grave, porém tais intervenções devem ser implementadas fielmente ao protocolo. A fidelidade refere-se à medida que uma intervenção adere ao modelo original e sua avaliação é essencial para que os desfechos possam ser creditados à intervenção. O objetivo deste estudo foi realizar uma revisão sistemática da literatura mundial das intervenções psicossociais destinadas a pacientes com transtornos mentais que possuem um instrumento ou método de avaliação de fidelidade ao modelo original. Métodos: Pesquisas bibliográficas sistemáticas foram realizadas para encontrar estudos relevantes ao tema nas seguintes bases de dados: Embase, Medline, Scopus e SciELO. Foram incluídos estudos de intervenções psicossociais, realizados na comunidade, dirigidos a pacientes diagnosticados com transtornos mentais. Intervenções de comparação poderiam incluir tanto o tratamento-padrão como uma intervenção de comparação ativa. A qualidade dos estudos foi avaliada de forma independente por dois revisores, utilizando critérios adaptados de instrumentos validados. Resultados: Trinta estudos preencheram os critérios de inclusão. Os estudos mostraram a eficácia da avaliação da fidelidade em diferenciar diferentes modelos de tratamento, sua validade preditiva para os desfechos e a confiabilidade dos instrumentos

\section{Palavras-chave}

Fidelidade, adesão ao protocolo, intervenção psicossocial, transtorno mental, revisão sistemática. utilizados, bem como os fatores facilitadores e os obstáculos para a obtenção de alta fidelidade nas intervenções avaliadas. Conclusão: Além de documentar a adesão ao modelo original, a fidelidade fornece informações relativas à população-alvo e aos desfechos esperados, o que contribui para que seja alcançada excelência no processo de implementação das intervenções psicossociais.

\section{ABSTRACT}

Objective: Psychosocial interventions have had a positive impact on the lives of people with severe mental illness. However, such interventions should be implemented accurately according to the protocol. Fidelity refers to the extent that an intervention adheres to the original model and its evaluation is essential so that the outcomes can be credited to the intervention. This study aims carry out a global systematic review of psychosocial interventions to patients suffering from mental illness which have an instrument or assessment method of fidelity to original model. Methods: Systematic literature searches were performed to find studies re-

1 Universidade Federal do Rio de Janeiro (UFRJ), Instituto de Estudos em Saúde Coletiva (IESC), Saúde Coletiva.

Recebido em

$15 / 6 / 2014$

Aprovado em
$31 / 8 / 2014$
Endereço para correspondência: Tatiana Fernandes Carpinteiro da Silva

Av. Horácio Macedo, s/n, llha do Fundão

21941-598 - Rio de Janeiro, RJ, Brasil

Telefone: (21)x

E-mail: tatiana_fcs@hotmail.com 


\section{Keywords}

Fidelity, protocol compliance, psychosocial interventions, mental disorders, systematic review. levant to the theme in the following databases: Embase, Medline, Scopus and SciELO. Studies of psychosocial intervention, conducted in community care for patients diagnosed with mental disorders were included. Comparison interventions could include either the standard treatment or intervention of active comparison. Study quality was assessed independently by two reviewers using criteria adapted from validated instruments. Results: Thirty studies met the inclusion criteria. Studies have shown the effectiveness of the assessment of fidelity to differentiate different treatment models, their predictive validity for outcomes and reliability of the instruments used, as well as facilitating factors and obstacles for achieving high fidelity in the evaluated interventions. Conclusion: More than documenting adherence to the original model, fidelity also provides information relating to the target population and the expected outcomes, which allows performing necessary adjustments during its course to be achieved excellence in the implementation process of such interventions.

\section{INTRODUÇÃO}

Os transtornos mentais estão entre as mais onerosas classes de doenças, por causa de sua alta prevalência, cronicidade, idade de início precoce e comprometimento resultante!. Atualmente, a Organização Mundial da Saúde (OMS) estima que cerca de 450 milhões de pessoas em todo o mundo sofrem de algum tipo de transtorno mental e que uma em cada quatro pessoas atendem aos seus critérios diagnósticos em algum momento de sua vida'.

Ao longo dos últimos anos, tornou-se claro que a farmacoterapia pode melhorar os sintomas dos transtornos mentais, mas tem efeito limitado sobre o funcionamento social e qualidade de vida, mesmo com adequada adesão à medicação. O tratamento ideal dos transtornos mentais graves deve incluir uma combinação de medicação e intervenções psicossociais, com orientação para a comunidade e envolvimento dos cuidadores ${ }^{1,2}$. No entanto, a grande proporção de pacientes não recebe esse atendimento abrangente, principalmente em países de baixa e média renda ${ }^{1,2}$.

Uma intervenção psicossocial pode ser definida como o conjunto de estratégias sociais e psicológicas que complementam o tratamento farmacológico e o gerenciamento de sintomas, cujo objetivo é melhorar o funcionamento social e pessoal, a qualidade de vida e a integração na comunidade de indivíduos que sofrem de transtornos mentais. Tem como foco a reabilitação desses pacientes no diz respeito ao desempenho de suas atividades habituais, aperfeiçoando suas habilidades pessoais e sociais, dando apoio para a execução de suas funções de modo mais ativo e independente na comunidade, além de melhorar a qualidade de vida do paciente e de sua família ${ }^{1-3}$. Há uma evidência crescente de pesquisa em saúde mental indicando que as intervenções psicossociais têm impacto positivo na vida das pessoas com transtorno mental grave, ajudando a melhorar suas capacidades pessoais e sociais em longo prazo e a promover a reintegração na comunidade ${ }^{3}$.

No entanto, a literatura sugere que a implementação de muitas dessas práticas tem falhado por vários motivos ${ }^{4}$.
Um deles é falta de um plano de implementação adequado, sem especificação clara do modelo e lacunas em relação à sua condução em diferentes locais ${ }^{4}$. O modelo de intervenção deverá especificar o tipo e a quantidade de intervenção que os pacientes vão receber, o seu modo de realização e a estrutura administrativa necessária para a implementação, além da qualificação dos profissionais envolvidos e o treinamento necessário para tanto. Além disso, a avaliação da fidelidade ao protocolo é essencial para que os resultados (positivos ou negativos) possam ser associados à intervenção ${ }^{5}$.

Fidelidade refere-se à medida que uma intervenção adere ao modelo original, incluindo características que são fundamentais para alcançar os resultados pretendidos e excluindo aqueles que possam interferir ${ }^{6}$. Medidas de fidelidade normalmente incluem uma combinação de indicadores qualitativos e quantitativos que avaliam o quanto os elementos-chave de uma intervenção foram implementados conforme o modelo original?

A avaliação da fidelidade tem utilidade para a clínica, para a pesquisa e para fins administrativos ${ }^{5,8}$ e baseia-se na relação entre a alta fidelidade e resultados desejados. De fato, estudos têm demonstrado que alta fidelidade está ligada a melhores resultados em muitas práticas baseadas em evidências. Porém, se a fidelidade ao modelo original não for assegurada, não se pode afirmar que os resultados obtidos são consequência daquela determinada intervenção $0^{9,10}$.

Muitas intervenções, especialmente aquelas que refletem a melhora na qualidade de vida ou em áreas de reabilitação, normalmente levam um longo tempo para mostrar resultados, não havendo retorno significativo em curto prazo da efetividade da intervenção. Em alguns aspectos, a medida da fidelidade pode servir como uma avaliação intermediária para a medida do resultado. Assim, avaliar se um programa está aderindo ao modelo pode fornecer informações preditivas sobre os resultados desejados?.

Os prestadores de serviços podem usar medidas de fidelidade, como uma medida clínica, comparando o trata- 
mento usual com a prática pretendida. Provedores do novo programa podem usar a medida de fidelidade como uma diretriz para assegurar a replicação, enquanto os programas existentes podem se referir a essas medidas para evitar desvio de protocolo no programa. Na pesquisa, a avaliação da fidelidade é fundamental, pois sem saber exatamente como a intervenção foi implementada é muito difícil atribuir mudanças ou diferenças em relação à prática usual ${ }^{11}$. A avaliação da fidelidade permite desconstruir o modelo para melhor identificar quais são os elementos críticos que conduzem aos resultados mais importantes. A fidelidade de modelos bem definidos fornece uma base conceitual para a adaptação e inovação, o que pode ser empiricamente estudado. Além disso, a avaliação da fidelidade é essencial em estudos multicêntricos, a fim de evitar descaracterização do modelo causada por diferenças culturais e entre profissionais ${ }^{9}$.

A avaliação da fidelidade nas intervenções psicossociais não apenas foca a conduta dos profissionais, mas também se dirige a aspectos estruturais do programa, como a qualificação dos profissionais envolvidos, a relação do número de pacientes por profissional, a localização dos serviços oferecidos, o material a ser utilizado pelos profissionais e a integração entre o tratamento usual e a intervenção proposta. É uma tarefa complexa, que envolve diversos aspectos e que indica quais elementos do modelo exercem papel crítico na obtenção dos resultados esperados ${ }^{5}$.

A avaliação da fidelidade de uma intervenção em saúde mental teve seus primórdios na psicoterapia. A importância de definir e mensurar elementos de um modelo proposto foi inicialmente reconhecida na década de 1960, quando pesquisas perceberam a impossibilidade de garantir a metodologia utilizada em seus estudos iniciais, já que não havia manuais de implementação específicos nem meios que avaliassem a qualidade dessa implementação ${ }^{6}$. Do mesmo modo que as intervenções psicoterápicas no passado, a reabilitação psicossocial ainda carece de literatura descritiva de seus modelos e da avaliação de sua fidelidade.

Apesar de a importância da avaliação da fidelidade ser atualmente reconhecida, intervenções psicossociais que utilizem esse método em sua implementação ainda são escassas, sendo estimadas em 3,5\% em todo o mundo ${ }^{12}$. No Brasil e demais países da América Latina, nenhuma intervenção implementada possui sistema de avaliação de fidelidade estabelecido. Portanto, é fundamental que se aprofunde o conhecimento sobre esse tipo de método de avaliação para que futuras intervenções sejam implementadas seguindo todas as etapas preconizadas para sua realização.

Portanto, o presente estudo teve como objetivo realizar uma revisão sistemática da literatura mundial das intervenções psicossociais destinadas a pacientes com transtornos mentais que possuem um instrumento ou método de avaliação de fidelidade ao modelo original.

\section{MÉTODOS}

\section{Estratégia de busca}

Pesquisas bibliográficas sistemáticas foram realizadas para encontrar estudos relevantes com base nas seguintes bases de dados: Embase, Medline, Scopus e SciELO, dos quais o último se destinou principalmente à procura de estudos realizados na América Latina. Os estudos foram pesquisados utilizando as seguintes palavras-chave, contidas no DeCS (Descritores em Ciências da Saúde) e MeSH (Medical Subject Headings): "fidelidade", "adesão ao manual", "cumprimento do protocolo", "avaliação do processo" e "saúde mental" em português, "fidelity", "guideline adherence", "protocol compliance", "process assessment" e "mental health" em inglês, e "fidelidad", "adhesión a directriz", "cumplimiento del protocolo", "evaluación del proceso" e "salud mental" em espanhol. Outros artigos foram encontrados por meio de busca de referências e revisões anteriores (Figura 1).

\section{Seleção dos estudos}

Foram considerados para inclusão os estudos de intervenções psicossociais, realizados na comunidade, dirigidas a pacientes diagnosticados com transtornos mentais. Intervenções de comparação poderiam incluir tanto o tratamento usualmente oferecido comparado à intervenção estudada como a comparação entre duas intervenções. Os participantes poderiam ser crianças, jovens ou adultos com transtornos mentais de início recente ou com a doença crônica. Os artigos deveriam ser publicados em periódicos nos idiomas português, inglês, ou espanhol. Os artigos devem ter como escopo a avaliação da fidelidade da intervenção por um instrumento ou método específico (Figura 1). Dois revisores avaliaram independentemente todos os artigos potencialmente relevantes para a inclusão. Divergências foram resolvidas por meio do diálogo entre os autores.

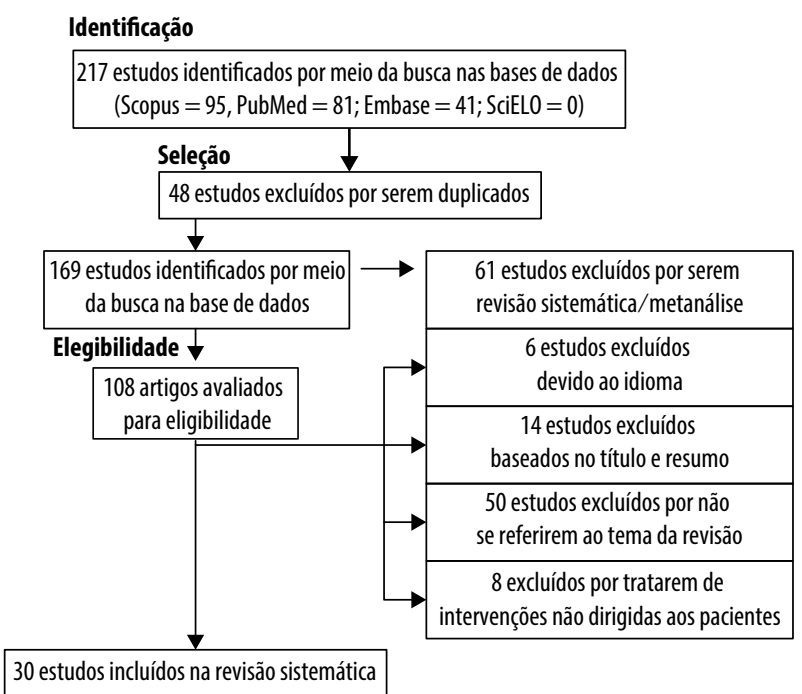

Figura 1. Diagrama de fluxo de seleção dos estudos. 


\section{Extração de dados}

Dois revisores extraíram independentemente os dados relevantes dos estudos incluídos em uma tabela que continha autores, local, ano, tipo de intervenção, população-alvo, duração das intervenções, objetivos da intervenção, instrumento utilizado para avaliar a fidelidade e principais resultados. Discrepâncias foram resolvidas por consenso entre os autores. Publicações duplicadas foram excluídas, bem como vários artigos sobre o mesmo estudo.

\section{Avaliação da qualidade dos estudos incluídos}

A qualidade dos estudos incluídos foi avaliada de forma independente por dois revisores, utilizando critérios adaptados de instrumento validado. O Critical Appraisal Checklist for Included Studies' ${ }^{13}$ considera de qualidade satisfatória para inclusão na revisão sistemática os estudos que obtiverem $\geq 50 \%$ da pontuação do cheklist. As pontuações foram comparadas e discordâncias foram resolvidas por consenso entre os revisores antes de atribuir uma pontuação final.

A busca nas bases de dados Medline, Embase, Scopus e SciELO encontrou um total de 217 citações. Após o ajuste para duplicatas, permaneceram 169 artigos. Desses, 61 foram excluídos, por serem revisão sistemática/metanálise e 14 estudos foram descartados porque, depois de se analisarem os títulos/resumos, eles claramente não cumpriam os critérios de inclusão. Estudos adicionais (50) foram descartados porque não avaliavam a fidelidade. Seis estudos foram excluídos por serem escritos em outro idioma que não o inglês/espanhol/português (três italianos, dois franceses e um alemão). Oito estudos foram excluídos por avaliar a fidelidade de intervenções destinadas à família ou aos profissionais envolvidos no tratamento de pacientes com transtorno mental. Trinta estudos preencheram os critérios e tiveram sua qualidade avaliada. Todos foram incluídos na revisão sistemática (Quadro 1) por teram obtido pontuação $\geq 50 \%$ no checklist de avaliação da qualidade, com pontuações variando de $57,1 \%$ a $100 \%$.

Quadro 1. Estudos que utilizaram método de avaliação da fidelidade

\begin{tabular}{|c|c|c|c|c|c|}
\hline $\begin{array}{l}\text { Autor/Ano/Local } \\
\text { do estudo }\end{array}$ & Intervenção & Objetivo da intervenção & População-alvo & $\begin{array}{l}\text { Método usado para avaliar a } \\
\text { fidelidade }\end{array}$ & Resultados \\
\hline $\begin{array}{l}\text { Teague et al. }{ }^{8} \text {, } \\
\text { 1998, EUA }\end{array}$ & $\begin{array}{l}\text { Tratamento Assertivo } \\
\text { Comunitário (ACT) }\end{array}$ & $\begin{array}{l}\text { Tratamento de base } \\
\text { comunitária alternativo } \\
\text { ao cuidado hospitalar que } \\
\text { auxilia na adaptação à vida } \\
\text { em sociedade }\end{array}$ & $\begin{array}{l}\text { Pacientes com transtorno } \\
\text { mental grave }\end{array}$ & $\begin{array}{l}\text { Escala Dartmouth ACT } \\
\text { (DACTS) }\end{array}$ & $\begin{array}{l}\text { A DACT foi capaz de distinguir programas em } \\
\text { diferentes niveis de fidelidade do ACT. Mostrou } \\
\text { evidências preliminares de validade preditiva }\end{array}$ \\
\hline $\begin{array}{l}\text { McHugo et } \\
\text { al. }{ }^{10}, 1999, \\
\text { EUA }\end{array}$ & $\begin{array}{l}\text { Tratamento Assertivo } \\
\text { Comunitário (ACT) }\end{array}$ & $\begin{array}{l}\text { Tratamento de base } \\
\text { comunitária alternativo } \\
\text { ao cuidado hospitalar que } \\
\text { auxilia na adaptação à vida } \\
\text { em sociedade }\end{array}$ & $\begin{array}{l}\text { Pacientes com transtorno } \\
\text { mental grave }\end{array}$ & $\begin{array}{l}\text { Lista com } 9 \text { componentes } \\
\text { essenciais do ACT e } 4 \\
\text { componentes essenciais do } \\
\text { programa de transtornos } \\
\text { duais }\end{array}$ & $\begin{array}{l}\text { Pacientes em programas ACT de alta fidelidade } \\
\text { apresentaram maior redução no uso de álcool } \\
\text { e drogas, maiores taxas de continuidade ao } \\
\text { tratamento e menos admissões hospitalares do } \\
\text { que aqueles em programas de baixa fidelidade }\end{array}$ \\
\hline $\begin{array}{l}\text { Ablon e } \\
\text { Jones }^{14}, 1999, \\
\text { EUA }\end{array}$ & $\begin{array}{l}\text { Psicoterapia } \\
\text { interpessoal (IPT) e } \\
\text { terapia cognitivo- } \\
\text { comportamental (TCC) }\end{array}$ & $\begin{array}{l}\text { Fornecer apoio a indivíduos } \\
\text { com depressão }\end{array}$ & $\begin{array}{l}\text { Pacientes ambulatoriais em } \\
\text { tratamento para transtorno } \\
\text { depressivo maior }\end{array}$ & $\begin{array}{l}\text { Processo de Psicoterapia } \\
\text { Q-Set (PQS) }\end{array}$ & $\begin{array}{l}\text { As sessões de ambas as terapias mostraram } \\
\text { mais fidelidade ao protótipo ideal da TCC. Além } \\
\text { disso, a fidelidade maior à TCC se correlacionou } \\
\text { mais positivamente com medidas de resultados } \\
\text { em ambas as modalidades terapêuticas }\end{array}$ \\
\hline $\begin{array}{l}\text { Carroll et al. }{ }^{15} \\
2000, \text { EUA }\end{array}$ & Psicoterapia & $\begin{array}{l}\text { Tratamento psicoterapêutico } \\
\text { comportamental para } \\
\text { transtornos por uso de } \\
\text { substâncias }\end{array}$ & $\begin{array}{l}\text { Pacientes com transtornos } \\
\text { por uso de substâncias }\end{array}$ & $\begin{array}{l}\text { Escala Yale de Aderência e } \\
\text { Competência (YACS) }\end{array}$ & $\begin{array}{l}\text { A validação da YACS indicou boa confiabilidade } \\
\text { e validade. Terapeutas tendem a superestimar } \\
\text { seus níveis de fidelidade quando comparados a } \\
\text { avaliadores independentes }\end{array}$ \\
\hline $\begin{array}{l}\text { Macias et al.16, } \\
\text { 2001, EUA }\end{array}$ & Modelo Clubhouse & $\begin{array}{l}\text { Promover a dignidade, } 0 \\
\text { contato social e a confiança } \\
\text { interpessoal necessária } \\
\text { para restabelecer a vida em } \\
\text { comunidade }\end{array}$ & $\begin{array}{l}\text { Pacientes com transtorno } \\
\text { mental grave }\end{array}$ & $\begin{array}{l}\text { Clubhouse Research and } \\
\text { Evaluation Screening Survey } \\
\text { (CRESS) }\end{array}$ & $\begin{array}{l}\text { A CRESS mostrou boa validade e ser de fácil } \\
\text { administração. Participantes de programas com } \\
\text { alta fidelidade tiveram taxas de emprego duas } \\
\text { vezes superiores aos dos demais pacientes }\end{array}$ \\
\hline $\begin{array}{l}\text { Salyers et al.", } \\
\text { 2003, EUA }\end{array}$ & $\begin{array}{l}\text { Tratamento Assertivo } \\
\text { Comunitário (ACT) }\end{array}$ & $\begin{array}{l}\text { Tratamento de base } \\
\text { comunitária alternativo } \\
\text { ao cuidado hospitalar que } \\
\text { auxilia na adaptação à vida } \\
\text { em sociedade }\end{array}$ & $\begin{array}{l}\text { Pacientes com transtorno } \\
\text { mental grave }\end{array}$ & $\begin{array}{l}\text { Escala Dartmouth ACT } \\
\text { (DACTS) }\end{array}$ & $\begin{array}{l}\text { A DACTS demonstrou utilidade em registrar } \\
\text { o quanto um programa segue os princípios } \\
\text { do ACT. Entretanto, gestores alegam falta de } \\
\text { perspectiva prática do instrumento devido } \\
\text { à falta de um ponto de corte para avaliar se } \\
\text { um programa preenche os critérios de modo } \\
\text { dicotômico }\end{array}$ \\
\hline $\begin{array}{l}\text { Hayes et al. }{ }^{18} \text {, } \\
\text { 2003, EUA }\end{array}$ & Projeto MISA & $\begin{array}{l}\text { Oferecer serviços que } \\
\text { melhorem o nível de } \\
\text { funcionamento de pacientes } \\
\text { com transtornos mentais e } \\
\text { uso de substâncias }\end{array}$ & $\begin{array}{l}\text { Pacientes com transtorno } \\
\text { mental grave comórbido ao } \\
\text { uso de substâncias }\end{array}$ & $\begin{array}{l}\text { A medida da fidelidade foi } \\
\text { realizada por meio da revisão } \\
\text { dos prontuários }\end{array}$ & $\begin{array}{l}\text { A avaliação foi feita considerando indicadores } \\
\text { selecionados por serem críticos ao bom } \\
\text { funcionamento do programa. Fidelidade foi } \\
\text { observada em } 72 \% \text { dos pacientes avaliados }\end{array}$ \\
\hline
\end{tabular}


Quadro 1. Estudos que utilizaram método de avaliação da fidelidade

\begin{tabular}{|c|c|}
\hline $\begin{array}{l}\text { Autor/Ano/Local } \\
\text { do estudo }\end{array}$ & Intervenção \\
\hline $\begin{array}{l}\text { Fiander } \\
\text { et al. }{ }^{19}, 2003 \text {, } \\
\text { Reino Unido }\end{array}$ & $\begin{array}{l}\text { Tratamento Assertivo } \\
\text { Comunitário }(\mathrm{ACT})\end{array}$ \\
\hline $\begin{array}{l}\text { Oshima } \\
\text { et al. }{ }^{20}, 2004, \\
\text { Japão }\end{array}$ & $\begin{array}{l}\text { Gerenciamento de caso } \\
\text { (case management) }\end{array}$ \\
\hline $\begin{array}{l}\text { McGrew e } \\
\text { Griss }^{21}, 2005 \text {, } \\
\text { EUA }\end{array}$ & $\begin{array}{l}\text { Programa de apoio ao } \\
\text { emprego (SE) }\end{array}$ \\
\hline $\begin{array}{l}\text { Falloon et al }^{22} \text {, } \\
2005, \text { Estudo } \\
\text { Multicêntrico }\end{array}$ & $\begin{array}{l}9 \text { intervenções (Goal } \\
\text { and problem oriented } \\
\text { assessment; Medication } \\
\text { strategies; Assertive } \\
\text { case management; } \\
\text { Mental health education; } \\
\text { Caregiver-based } \\
\text { problem solving; } \\
\text { Living skills training; } \\
\text { psychosocial strategies } \\
\text { for residual problems; } \\
\text { Crisis prevention and } \\
\text { intervention; Booster } \\
\text { sessions) }\end{array}$ \\
\hline
\end{tabular}

Essock et al. ${ }^{23}$, Terapia cognitivo2006, EUA comportamental (TCC)

Milby et al. ${ }^{24}$, Intervenção multimodal 2007, EUA

Van Voorhees Intervenção on-line et al. ${ }^{25}, 2007$, para prevenção de USA depressãousando terapia cognitivocomportamental (TCC) e terapia interpessoal (IPT)

Bond et al. ${ }^{26}$, Programa de apoio ao 2008, EUA emprego(SE)

Brunette Tratamento Integrado et al. ${ }^{27}, 2008$, de Transtornos Duais EUA

\section{Objetivo da intervenção}

Tratamento de base

comunitária alternativo

ao cuidado hospitalar que

auxilia na adaptação à vida

em sociedade

Tratamento de base

comunitária alternativo

ao cuidado hospitalar que

auxilia na adaptação à vida em sociedade

\section{Melhorar desfechos} ocupacionais em pacientes com transtorno mental grave

Melhorar resultados em diferentes aspectos do tratamento de pacientes do espectro esquizofrênico cada intervenção com seu objetivo específico

Oferecer apoio a indivíduos com transtornos depressivos e de estresse pós-traumático após 0 atentado de 11 de setembro

\section{Melhorar desfechos em} população de rua usuária de substâncias psicoativas

Prevenir depressão maior em adultos jovens

Increasing employment opportunities for people diagnosed with mental health problems

Melhorar o funcionamento de pacientes com transtorno mental grave comórbido ao uso de substância psicoativa
Pacientes com transtorno mental grave comórbido ao uso de substâncias

Pacientes com transtorno
mental grave

Pacientes com transtorno mental grave

Pacientes com transtorno mental grave

Pacientes com transtorno mental grave

Indivíduos emocionalmente afetados pelo atentado de 11 de setembro

População de rua usuária de substâncias psicoativas

Adultos jovens (18 a 25 anos) com risco de desenvolver depressão maior

Pacientes com transtorno mental grave

Escala de fidelidade SE

Avaliação da fidelidade por meio de entrevistas semiestruturadas a cada 6 meses
Entrevista com os pacientes sobre os itens essenciais da TCC por telefone

Avaliação de vídeos usando de checklist comportamental

Checklist contendo os elementos essenciais do manual da intervenção
Crianças com distúrbios emocionais
Avaliação de supervisão clínica semanal por meio de formulários específicos
Questões curtas para os participantes se mostraram úteis e custo-efetivas na avaliação da fidelidade da intervenção

A avaliação de vídeos mostrou ser efetiva na medida da fidelidade de intervenções complexas

0 checklist foi satisfatório para avaliação da fidelidade das entrevistas bem como dos módulos on-line, com 100\% dos elementos essenciais conduzidos na intervenção 
Quadro 1. Estudos que utilizaram método de avaliação da fidelidade

\begin{tabular}{|c|c|c|c|c|c|}
\hline $\begin{array}{l}\text { Autor/Ano/Local } \\
\text { do estudo }\end{array}$ & Intervenção & Objetivo da intervenção & População-alvo & $\begin{array}{l}\text { Método usado para avaliar a } \\
\text { fidelidade }\end{array}$ & Resultados \\
\hline $\begin{array}{l}\text { Boyce e } \\
\text { Secker }{ }^{29} \\
\text { 2008, Reino } \\
\text { Unido }\end{array}$ & $\begin{array}{l}\text { Programa de apoio ao } \\
\text { emprego (SE) }\end{array}$ & $\begin{array}{l}\text { Aumentar a oportunidade de } \\
\text { emprego para pacientes com } \\
\text { transtorno mental grave }\end{array}$ & $\begin{array}{l}\text { Pacientes com transtorno } \\
\text { mental grave }\end{array}$ & Escala de fidelidade SE & $\begin{array}{l}\text { Baixa fidelidade foi associada a restrições } \\
\text { no orçamento e na política organizacional. } \\
\text { A escala de fidelidade SE se mostrou um } \\
\text { bom método para monitorar o processo de } \\
\text { implementação }\end{array}$ \\
\hline $\begin{array}{l}\text { Forsner et } \\
\text { al. }{ }^{30}, 2008, \\
\text { EUA }\end{array}$ & $\begin{array}{l}\text { Diretriz clínica para } \\
\text { o tratamento da } \\
\text { depressão em pacientes } \\
\text { suicidas }\end{array}$ & $\begin{array}{l}\text { Introdução de diretrizes } \\
\text { clínicas baseadas em } \\
\text { evidência para pacientes } \\
\text { deprimidos e com ideação } \\
\text { suicida }\end{array}$ & $\begin{array}{l}\text { Pacientes com depressão e } \\
\text { ideação suicida }\end{array}$ & $\begin{array}{l}\text { A prática recomendada } \\
\text { nas diretrizes clínicas foi } \\
\text { usada como indicador da } \\
\text { fidelidade, utilizando } 0 \\
\text { instrumento AUDIT }\end{array}$ & $\begin{array}{l}\text { A avaliação da fidelidade mostrou diferenciação } \\
\text { da prática usual tanto no tratamento da } \\
\text { depressão quanto no manejo do suicídio. A } \\
\text { fidelidade às diretrizes clínicas foi aumentada } \\
\text { por um ativo processo de implementação }\end{array}$ \\
\hline $\begin{array}{l}\text { Woltmannet } \\
\text { al. }{ }^{31}, 2008, \\
\text { EUA }\end{array}$ & $\begin{array}{l}5 \text { intervenções } \\
\text { (Integrated dual } \\
\text { disorders treatment, } \\
\text { supported employment, } \\
\text { family psychoeducation, } \\
\text { assertive community }\end{array}$ & $\begin{array}{l}\text { Melhorar o funcionamento } \\
\text { de pacientes com transtorno } \\
\text { mental grave em diferentes } \\
\text { aspectos, de acordo com } \\
\text { cada intervenção }\end{array}$ & $\begin{array}{l}\text { Pacientes com transtorno } \\
\text { mental grave }\end{array}$ & $\begin{array}{l}\text { Foram desenvolvidos } \\
\text { instrumentos de } \\
\text { avaliação da fidelidade } \\
\text { para cada intervenção, } \\
\text { operacionalizando a medida } \\
\text { dos componentes essenciais }\end{array}$ & $\begin{array}{l}\text { SE e ACT tiveram a fidelidade mais alta } \\
\text { durante os dois anos de avaliação. IMR e } \\
\text { IDDT apresentaram a menor fidelidade aos } \\
\text { programas. A alta rotatividade dos membros da } \\
\text { equipe foi um fator preditor de baixa fidelidade } \\
\text { ao longo do período avaliação }\end{array}$ \\
\hline
\end{tabular}

Catty et al. ${ }^{32}$, Programa de apoio ao Increasing employment 2008, Reino emprego (SE)-Apoio opportunities for people Unido ao posicionamento individual (IPS) diagnosed with mental health problems
Pacientes com transtorno mental grave
Escala de fidelidade SE e IPS

(1)

\author{
A prática recomendada \\ trizes clínicas foi \\ fidelidade, utilizando 0 \\ Foram desenvolvidos \\ para cada intervenção, \\ dos componentes essenciais \\ para a implementação
} ao longo do período avaliação

\section{Martino et Terapia de Valorização al. ${ }^{33}, 2008, \quad$ Motivacional (MET)} EUA

Wilson e Crisanti $^{34}$, 2009, EUA

ACT aplicado ao programa de Habitação Primeiro ("Housing First")
Aumentar a motivação do Pacientes com transtornos paciente dentro das sessões, por uso de substâncias sua continuidade (dias de tratamento) e melhorar os resultados em relação ao uso de substâncias

Melhorar o funcionamento de pacientes com transtorno mental grave comórbido ao uso de substância psicoativa
Pacientes com transtorno mental grave comórbido ao uso de substâncias
Escala de Avaliação Independente do Vídeo (IRTS)

Escala de Fidelidade do Tratamento de Transtornos Duais (DDTFS)
Promover acesso à habitação População de rua usuária e serviços que atendam às necessidades prioritárias dos pacientes de substâncias psicoativas e portadora de transtornos mentais
Escala Dartmouth do ACT (DACTS) - Avaliação por telefone

\section{Killaspy et al. ${ }^{36}, 2009$ \\ Tratamento Assertivo
Comunitário (ACT)} Reino Unido
Tratamento de base comunitária alternativo ao cuidado hospitalar que auxilia na adaptação à vida em sociedade
Pacientes com transtorno mental grave
Escala Dartmouth ACT (DACTS) - Avaliação por telefone
A maior pontuação na escala de fidelidade IPS promoveu maior magnitude de efeito a favor do modelo IPS. Além disso, a manutenção de alta fidelidade e orientação para competências relacionais se mostraram um foco importante para todas as intervenções de formação profissional, levando à melhora nos resultados de obtenção de empregos

A maior fidelidade foi relacionada à mudança positiva na motivação dos pacientes e ao percentual de testes negativos para rastreamento de drogas obtido durante a fase de tratamento de 4 semanas

A pontuação total da fidelidade não fez distinção entre o centro de saúde mental da comunidade e programas de diagnóstico especializados em transtornos duais, no entanto uma análise de cluster revelou validade concorrente com base na avaliação individual dos itens. As conclusões das avaliações individuais dos itens foram válidas, porém a validade das conclusões com base nos escores totais permanece sem fundamento

Os desvios do modelo ACT observados foram atribuídos à escolha do paciente inerente ao programa Habitação Primeiro e aos aspectos dessa população, que está mais desconectada de apoios sociais do que outros indivíduos com doença mental, devido a suas histórias de longa permanência nas ruas e maior envolvimento com abuso de substâncias

0 estudo randomizado de ACT realizado na Inglaterra não encontrou nenhuma vantagem clinicamente significativa sobre os cuidados habituais de equipes de saúde mental da comunidade. No entanto, os clientes ACT estavam mais satisfeitos e mais engajados com os serviços. Estudos europeus da ACT mostraram menor eficácia clínica e custoefetividade do que nos EUA. As explicações incluem baixa fidelidade ao modelo ACT em alguns centros e elevados padrões de serviços de saúde mental comunitária. ACT foi mais bem-sucedido do que os serviços locais na continuidade dos pacientes mais difíceis 
Quadro 1. Estudos que utilizaram método de avaliação da fidelidade

\begin{tabular}{|c|c|c|c|c|c|}
\hline $\begin{array}{l}\text { Autor/Ano/Local } \\
\text { do estudo }\end{array}$ & Intervenção & Objetivo da intervenção & População-alvo & $\begin{array}{l}\text { Método usado para avaliar a } \\
\text { fidelidade }\end{array}$ & Resultados \\
\hline $\begin{array}{l}\text { Long et al. }{ }^{37} \text {, } \\
2010 \text {, EUA }\end{array}$ & $\begin{array}{l}\text { Intervenção cognitivo- } \\
\text { comportamental } \\
\text { multicomponentes para } \\
\text { transtorno de estresse } \\
\text { pós-traumático (TEPT) }\end{array}$ & $\begin{array}{l}\text { Aliviar sintomas de TEPT nos } \\
\text { pacientes com transtornos } \\
\text { mentais graves }\end{array}$ & $\begin{array}{l}\text { Pacientes com transtornos } \\
\text { mentais graves em } \\
\text { comorbidade ao TEPT }\end{array}$ & $\begin{array}{l}\text { Formulário de Avaliação de } \\
\text { Adesão e Competência do } \\
\text { terapeuta (TAC) }\end{array}$ & $\begin{array}{l}\text { A psicoterapia cognitivo-comportamental } \\
\text { individualizada para pacientes com transtorno } \\
\text { mental grave e TEPT não compromete a } \\
\text { fidelidade dos terapeutas para instruir de } \\
\text { forma eficaz e orientar os pacientes por meio } \\
\text { de sessão de exercícios (incluindo exposição } \\
\text { imaginária), fornecer um retorno adequado } \\
\text { aos pacientes e rever, apresentar e explicar as } \\
\text { tarefas de casa }\end{array}$ \\
\hline
\end{tabular}

\begin{tabular}{|c|c|c|c|c|c|}
\hline $\begin{array}{l}\text { McGrew et } \\
\text { al. }^{38}, 2011, \\
\text { EUA }\end{array}$ & $\begin{array}{l}\text { Tratamento Assertivo } \\
\text { Comunitário (ACT) }\end{array}$ & $\begin{array}{l}\text { Tratamento de base } \\
\text { comunitária alternativo } \\
\text { ao cuidado hospitalar que } \\
\text { auxilia na adaptação à vida } \\
\text { em sociedade }\end{array}$ & $\begin{array}{l}\text { Pacientes com transtorno } \\
\text { mental grave }\end{array}$ & $\begin{array}{l}\text { Escala Dartmouth ACT } \\
\text { (DACTS) - Avaliação por } \\
\text { telefone }\end{array}$ & $\begin{array}{l}\text { A avaliação da fidelidade ao ACT por telefone } \\
\text { mostrou-se válida e confiável. A experiência } \\
\text { prévia do avaliador da fidelidade não } \\
\text { teve impacto significante na validade e } \\
\text { confiabilidade }\end{array}$ \\
\hline $\begin{array}{l}\text { Monroe- } \\
\text { DeVita et al. }{ }^{9} \text {, } \\
\text { 2011, EUA }\end{array}$ & $\begin{array}{l}\text { Tratamento Assertivo } \\
\text { Comunitário (ACT) }\end{array}$ & $\begin{array}{l}\text { Tratamento de base } \\
\text { comunitária alternativo } \\
\text { ao cuidado hospitalar que } \\
\text { auxilia na adaptação à vida } \\
\text { em sociedade }\end{array}$ & $\begin{array}{l}\text { Pacientes com transtorno } \\
\text { mental grave }\end{array}$ & $\begin{array}{l}\text { Instrumento para avaliação } \\
\text { da ACT (TMACT) }\end{array}$ & $\begin{array}{l}\text { A escala TMACT apresentou padrões mais } \\
\text { elevados de desempenho mediante avaliação } \\
\text { melhorada da recuperação de orientação e } \\
\text { trabalho em equipe, além de ser mais sensível a } \\
\text { mudanças do que a escala DACTS }\end{array}$ \\
\hline $\begin{array}{l}\text { Aarons et al. }{ }^{39}, \\
\text { 2012, EUA }\end{array}$ & Autocuidado (SC) & $\begin{array}{l}\text { Visitas domiciliares para } \\
\text { a preservação da família } \\
\text { e reunificação do sistema } \\
\text { de serviços de bem-estar } \\
\text { da criança, a fim de reduzir } \\
\text { negligência }\end{array}$ & $\begin{array}{l}\text { Crianças sofrendo } \\
\text { negligência }\end{array}$ & $\begin{array}{l}\text { Monitoramento estruturado } \\
\text { da fidelidade como consulta } \\
\text { permanente para os } \\
\text { prestadores de serviços }\end{array}$ & $\begin{array}{l}\text { A fidelidade foi associada significativamente } \\
\text { a maior retenção dos profissionais nessa } \\
\text { implementação. No entanto, alguns membros } \\
\text { da equipe consideraram o monitoramento de } \\
\text { fidelidade um procedimento invasivo }\end{array}$ \\
\hline $\begin{array}{l}\text { Randall et } \\
\text { al. }{ }^{40}, 2012, \\
\text { Canadá }\end{array}$ & $\begin{array}{l}\text { Tratamento Assertivo } \\
\text { Comunitário (ACT) }\end{array}$ & $\begin{array}{l}\text { Tratamento de base } \\
\text { comunitária alternativo } \\
\text { ao cuidado hospitalar que } \\
\text { auxilia na adaptação à vida } \\
\text { em sociedade }\end{array}$ & $\begin{array}{l}\text { Pacientes com transtorno } \\
\text { mental grave }\end{array}$ & $\begin{array}{l}\text { Instrumento para avaliação } \\
\text { da fidelidade ACT de Ontário } \\
\text { - Avaliação por telefone }\end{array}$ & $\begin{array}{l}\text { Nenhum dos Coordenadores ACT percebe } \\
\text { seu programa como totalmente compatível } \\
\text { com todos os padrões do modelo. Dado o } \\
\text { crescente corpo de pesquisa que demonstra } \\
\text { uma associação entre a fidelidade às normas } \\
\text { do programa e a melhora do desfecho } \\
\text { para os pacientes, essa baixa fidelidade } \\
\text { relatada levanta sérias preocupações sobre } \\
\text { o funcionamento dos programas e até que } \\
\text { ponto os benefícios do modelo ACT estão sendo } \\
\text { obtidos no Canadá }\end{array}$ \\
\hline
\end{tabular}

\section{RESULTADOS}

A maioria dos estudos que avaliava a fidelidade foi realizada nos Estados Unidos (EUA) (76,6\%), seguida pelo Reino Unido (10\%). Todos foram escritos no idioma inglês. Não foram encontrados estudos realizados na América Latina. Os estudos incluídos foram realizados entre os anos de 1998 e 2012. As intervenções que tiveram a fidelidade avaliada em mais estudos foram: Tratamento Comunitário Assertivo (ACT - Assertive Community Treatment; 11 estudos), Apoio ao Emprego SE (cinco estudos) e Tratamento Integrado de Transtornos Duais (IDDT - Integrated Dual Disorders Treatment; três estudos).

A população-alvo dessas intervenções foi, em sua maioria, pacientes com transtornos mentais graves (53,3\%) e pacientes com transtornos mentais graves com uso de substâncias psicoativas comórbidas (16,7\%), seguidos por pacientes com transtorno por uso de substâncias (10\%), pacientes com transtornos depressivo-ansiosos (13,3\%) e crianças com algum tipo de transtorno comportamental (6,7\%).

As intervenções tiveram duração variando de 3 a 22 seções naqueles em que a duração foi descrita $(16,7 \%)$, sendo ilimitada na maioria das intervenções (33,3\%), e não tiveram a duração especificada no artigo em 50\% dos casos.

A maioria dos estudos utilizou um método específico na avaliação da fidelidade, como escalas ou cheklists (90\%). Apenas três estudos utilizaram avaliações subjetivas, como entrevistas aos pacientes e análise de prontuários ou de vídeos.

Os estudos incluídos se referiam apenas à avaliação de fidelidade de uma intervenção em 83,3\% dos casos, enquanto o restante dos estudos se referia ao desenvolvimento de instrumentos de avaliação de fidelidade ou associavam esses dois objetivos (16,7\% dos casos).

De modo geral, os estudos mostraram a eficácia da avaliação da fidelidade em diferenciar diferentes modelos de tratamento, sua validade preditiva para os desfechos, a confiabilidade dos instrumentos utilizados nessa avaliação e de suas variações, bem como os fatores facilitadores e os obstáculos para a obtenção de alta fidelidade nas intervenções avaliadas.

Todos os estudos analisados qualitativamente foram incluídos na revisão, por terem obtido pontuação $\geq 50 \%$ no Critical Appraisal Checklist for Included Studies, variando de 
$57,1 \%$ a $100 \%$. Os estudos que apresentaram maior pontuação ${ }^{19,35,36}$ utilizaram instrumento validado em suas avaliações, definiram com clareza seus objetivos e critérios de inclusão e exclusão, discutiram os pontos positivos e limitações do estudo e detalharam seus métodos de coleta de dados, população-alvo e local de realização do estudo. Os estudos de menor pontuação não preencheram esses quesitos de modo satisfatório ${ }^{14,18,22}$.

\section{DISCUSSÃO}

Os esforços de construção de um modelo de intervenção psicossocial foram iniciados nos EUA no final da década de 1970. Considerando que a maioria das intervenções psicossociais existentes teve seu modelo construído recentemente nos EUA e que lá também foi iniciada a cultura da prática baseada em evidência, é natural que o desenvolvimento e o estudo de métodos de avaliação da fidelidade tenham sua origem e maior aplicação neste país, $8,17,39$.

O modelo de intervenção que teve sua fidelidade mais avaliada foi o tratamento ACT. Essa intervenção foi originalmente desenvolvida e implementada nos EUA como forma de ajudar as pessoas com transtorno mental grave a funcionarem mais plenamente na comunidade, reduzindo internações hospitalares e melhorando a qualidade de suas vidas. Tem como característica a utilização de equipes multidisciplinares que fornecem serviços de tratamento e apoio adaptados individualmente às necessidades dos pacientes. A maioria dos serviços é prestada fora dos locais usuais de atendimento à saúde mental, incentivando a integração na comunidade. Desde os anos 1980, o modelo ACT foi replicado em vários locais, como Estados Unidos, Europa e Canadá, e estudos têm demonstrado bons resultados relacionados à diminuição de taxas de hospitalização, melhor qualidade de vida e altos níveis de satisfação do cliente e da família 8,10,31,40. Também por ser uma das intervenções mais conduzidas, possui mais de um método de medida da fidelidade, incluindo mais de uma escala de avaliação (DACTS, TMACT, Ontario ACT Program Fidelity Too) e variações do meio de obtenção dos dados (aplicação das escalas por telefone) 9,35,36,38,40.

O modelo de Apoio ao Emprego (SE - Supported Emplyment), também desenvolvido nos EUA na década de 1980, visa ajudar a apoiar pessoas com transtornos mentais a conseguirem e manterem empregos competitivos em ambientes integrados. Tem sido conduzido em diferentes países, demonstrando algumas variações nos resultados obtidos de acordo com a organização e políticas de implementação locais. Possui dois instrumentos de avaliação da fidelidade (SE Fidelity Scale e QSEIS) 21,26,28. O IDDT oferece serviços para o tratamento da comorbidade entre transtornos mentais graves e o transtorno por uso de substâncias em um tratamento abrangente. Aproximadamente metade das pessoas com doença mental grave apresentará transtorno por uso de substância comórbido (abuso ou dependência) em algum momento de sua vida ${ }^{27}$. Pessoas com distúrbios duais têm pior prognóstico do que aqueles com um só transtorno. Comparado às demais intervenções, o tratamento integrado de transtornos duais foi uma das práticas que mais foram executadas com baixa fidelidade ${ }^{31,34}$. Talvez isso se associe ao fato de que essa intervenção não tem se mostrado consistentemente superior em comparação com os tratamentos usuais para essa população em todos os estudos, necessitando de mais pesquisas a respeito. Também deve ser considerado o fato de que o IDDT é uma intervenção complexa, que envolve múltiplos componentes e requer grande número de modificações nos provedores, na organização e no ambiente dos serviços. Por esses motivos, talvez seja uma intervenção mais difícil de ser implementada do que as demais intervenções com população-alvo com único diagnóstico 27,31.

De acordo com os estudos observados, as intervenções psicossociais que avaliam sua fidelidade são dirigidas principalmente a pacientes com transtornos mentais graves, tanto como único diagnóstico $(53,3 \%)$ como em comorbidade aos transtornos por uso de substâncias psicoativas (16,7\%). Tal fato pode estar relacionado à cronicidade de tais transtornos e ao prejuízo no funcionamento social desses pacientes, que requerem atenção especial e intervenções conduzidas sob condições otimizadas, a fim de se conseguir os resultados esperados. Além disso, sabe-se que, embora a farmacoterapia tenha papel fundamental na melhora dos sintomas dessa população, deixa a desejar no que diz respeito ao ajustamento social e à qualidade de vida ${ }^{8,9,20,40}$.

Os pacientes com transtornos depressivo-ansiosos (representando 13,3\% dos estudos) foram tratados predominantemente com intervenções psicoterápicas, como terapia cognitivo-comportamental e terapia interpessoal. Nem todos os estudos demonstraram diferenciação entre as intervenções propostas por meio da avaliação da fidelidade $14,23,25,30$. Intervenções psicossociais foram usadas em menor proporção em pacientes com transtorno por uso de substâncias psicoativas (10\%) e crianças com algum tipo de transtorno comportamental (6,7\%). Em ambos os casos, foram utilizados diferentes tipos de intervenção e métodos de avaliação a fidelidade, com resultados positivos na associação entre fidelidade e resultados esperados da intervenção $15,24,28,33,40$.

A avaliação da fidelidade é útil no acompanhamento longitudinal das intervenções, a fim de que a adesão ao protocolo não se perca ao longo do tempo, principalmente quando ocorrem mudanças inesperadas na organização e nos profissionais envolvidos ${ }^{31,39}$. Isso é importante tanto nas intervenções curtas, a fim de que a repetição dos ciclos não caia na rotina e acabe levando à negligência de determinados procedimentos, como também nas intervenções de longa duração, para que o efeito do passar do tempo não 
acabe direcionando os profissionais à realização das práticas de tratamento anteriores à intervenção. Alguns estudos observaram fidelidade crescente ao longo do primeiro ano de implementação, com diminuição da fidelidade no segundo ano ${ }^{4,26,31}$. Isso pode ocorrer devido à aquisição de experiência prática no modelo durante o primeiro ano e algum descuido na realização dos procedimentos ao se afastar do período de implementação, no qual foram realizados os treinamentos nos procedimentos necessários à intervenção. Daí a importância da avaliação da fidelidade também como um alerta para a realização de novo treinamento da equipe profissional quando indicado. Nesta revisão, apenas 16,7\% dos estudos especificaram a duração da intervenção proposta ${ }^{14,23,25,33,37}$. Muitas intervenções têm duração ilimitada, o que pode implicar não apenas cuidado permanente em manter fidelidade, bem como custos necessários para tal monitoramento.

A fidelidade de uma intervenção pode ser medida por diferentes métodos. Idealmente, envolveria a associação de estratégias quantitativas e qualitativas, a fim de que hipóteses sejam geradas, contextualizadas e testadas, para aprofundar o conhecimento sobre quais elementos são importantes no processo de implementação bem-sucedido ${ }^{39}$. Os meios de avaliação da fidelidade mais comuns são as escalas modelo -específicas 8,19,21,32-34, porém checklists com os elementos-chave da intervenção, entrevistas com profissionais ou pacientes, grupos focais, consulta aos prontuários, análise de vídeos da intervenção no dia a dia também são utilizados ${ }^{11,23-25,28}$. Métodos mais baratos para avaliar a fidelidade têm se mostrado efetivos, como a aplicação das escalas ou entrevistas por telefone $e^{5,6,23,38,40}$. Nesta revisão, apenas três estudos não utilizaram instrumentos específicos ${ }^{10,18,23}$ Recomenda-se que a fidelidade seja avaliada por um avaliador independente.

Estudos mostraram que, se o avaliador da fidelidade é um dos profissionais envolvidos na intervenção, a fidelidade é superestimada. A fim de evitar vieses na avaliação da fidelidade, é indicada a seleção de profissionais não envolvidos diretamente na implementação e que recebam treinamento apropriado no instrumento e nos métodos de avaliação. Esse instrumento, por sua vez, deve ter sido devidamente testado e possuir boas propriedades psicométricas para que a fidelidade seja adequadamente avaliada $a^{5,15}$.

O fato de os métodos não fornecerem resultados de forma dicotômica, ou seja, sem um ponto de corte estabelecido que auxiliasse a avaliação do gestor, foi criticado por órgãos financiadores das intervenções ${ }^{27}$. A falta de congruência entre a pontuação das escalas de fidelidade, a implementação e o tipo de intervenção poderia representar falência da escala de fidelidade, mas também pode representar erro de critério na escolha dos itens a serem considerados na avaliação de determinado modelo. Na ausência de um padrão-ouro, a utilização de múltiplos critérios de avaliação poderia reforçar a validade em estudos futuros. Mais estudos devem ser conduzidos a fim de que pontos de corte possam ser estabele- cidos nos instrumentos de avaliação da fidelidade. A medida da fidelidade é útil para monitorar a qualidade da implementação financiada nos diferentes centros, por exemplo, comparando regiões (urbanas $x$ rurais), centros individuais com a média geral e comparando a manutenção da fidelidade da implementação no decorrer do tempo $0^{10,34}$.

A avaliação da fidelidade de uma intervenção é especialmente importante em estudos multicêntricos, nos quais alguns resultados podem ser mais prevalentes em alguns sites do que em outros. O fato de alguns programas se distanciarem do modelo original por causa de diferenças culturais, diferenças na clientela atendida ou até mesmo características do tratamento usual em que a intervenção está sendo implementada pode ser a explicação, daí a importância de avaliar a fidelidade nos diferentes centros para que diferenças nos resultados possam ser interpretadas ${ }^{27,31,34}$. Estudos mostraram que a mesma intervenção - ACT - realizada nos EUA e no Reino Unido mostrou diferentes desfechos para os pacientes, mesmo quando comparados centros que conduziram a intervenção com fidelidade adequada ao modelo proposto. A diminuição nas internações dos participantes da ACT nos EUA não ocorreu naqueles submetidos à intervenção no Reino Unido, onde não foram encontradas vantagens da ACT em relação ao tratamento usual. Explicações incluem o fato de o Reino Unido já ter como tratamento usual um modelo efetivo, o qual não necessitaria ser complementado com esse tipo de intervenção, e a baixa fidelidade encontrada em alguns dos centros que conduziram a intervenção ${ }^{36}$. No Canadá, a fidelidade ao modelo ACT foi baixa, bem como o uso de métodos de avaliação da fidelidade. Tal fato leva ao questionamento sobre se a própria avaliação periódica da fidelidade não poderia atuar como um fator independente no aumento da qualidade, já que o simples fato de saber que serão avaliados recorrentemente faria com que os profissionais envolvidos se empenhassem mais na condução adequada dos procedimentos ${ }^{40}$. Em relação à clientela atendida, a fidelidade da ACT não foi satisfatória quando essa intervenção foi utilizada em população de rua com transtornos mentais. Esse desvio do modelo foi atribuído aos aspectos peculiares de tal população, por serem mais desconectados de apoios sociais do que outros indivíduos com transtorno mental em decorrência de sua longa permanência nas ruas e da maior prevalência de abuso de substâncias ${ }^{35}$.

Diversos estudos têm associado a maior fidelidade ao protocolo a melhores desfechos da intervenção. Tais associações podem ajudar na identificação de elementos críticos preditores de resultados para os pacientes. Essa aplicação está relacionada à validade preditiva da avaliação da fidelidade $^{5}$. Diversos estudos mostraram que alta fidelidade a ACT resultou em menor taxa de hospitalização, melhores desfechos clínicos e maior satisfação com o tratamento ${ }^{19,40}$. No tratamento de transtorno mental comórbido ao uso de 
substâncias, alta fidelidade ao ACT mostrou maior redução no uso de substâncias e manteve maiores taxas de remissão do que em pacientes de programas com baixa fidelidade 9 . Pacientes da intervenção Clubhouse com alta fidelidade tiveram taxas de emprego duas vezes maior do que aqueles que participavam de programas com baixa fidelidade ${ }^{16}$. Na terapia de aumento motivacional (MET - motivational enhancement therapy), a fidelidade foi relacionada positivamente à mudança na motivação do cliente. Também se mostrou associada ao percentual de triagem de drogas negativo obtido durante a fase de tratamento de quatro semanas ${ }^{33}$. Esses e outros exemplos na literatura reforçam a crescente importância da fidelidade como preditora do desfecho em intervenções psicossociais.

A fidelidade ao modelo é reflexo da qualidade do processo de implementação da intervenção. Estudos mostram que quanto maior a fidelidade de uma intervenção, mais adequados foram os procedimentos de implementação ${ }^{22,29}$. Ou seja, a medida da fidelidade não apenas reflete a qualidade do trabalho após o início da intervenção, mas também todos os procedimentos realizados até esse momento.

Intervenções com alta fidelidade ao modelo têm mostrado associação com a retenção dos profissionais envolvidos. Estudos que seguem o protocolo têm tido continuidade do trabalho pela mesma equipe, o que é importante para a intervenção, já que alta rotatividade dos profissionais está associada a menor produtividade em curto e longo prazo, menor efetividade organizacional, interferência na qualidade e nos resultados dos serviços fornecidos, além de aumentar os custos com recrutamento e treinamento de novos membros $23,31,39$. Por outro lado, a própria mudança nos profissionais durante o processo pode levar à baixa qualidade da implementação ${ }^{31}$. Qualitativamente, alguns profissionais consideram a avaliação da fidelidade um procedimento invasivo, por terem sua conduta profissional julgada, apesar de reconhecidos os benefícios desse procedimento ${ }^{39}$.

A avaliação da fidelidade mostrou utilidade na diferenciação entre dois métodos de tratamento, ou seja, entre a intervenção e o tratamento usualmente fornecido ou entre dois tipos de intervenção ${ }^{8,14,26}$. A avaliação da fidelidade em um estudo de terapia cognitivo-comportamental e terapia interpessoal mostrou que, embora as intervenções tenham seus elementos-chave próprios, muitas características se sobrepõem nos modelos ${ }^{14}$. Algumas vezes, o método de avaliação da fidelidade não foi capaz de realizar tal distinção. Em um estudo de comparação entre o modelo de IDDT e o tratamento usual, o método de avaliação da fidelidade utilizado não foi capaz de diferenciar consistentemente os dois programas ${ }^{34}$. As diferenças substanciais nos programas do mesmo tipo, bem como a sobreposição entre os programas de diferentes tipos, têm sido documentadas 8,14,26,34

Diversos fatores foram associados à alta fidelidade em uma intervenção, entre eles a baixa rotatividade dos pro- fissionais no serviço, experiência da equipe, liderança administrativa competente, expertise do coordenador da intervenção, adequado treinamento da equipe nos aspectos fundamentais da intervenção, consultoria regular a um especialista na intervenção, material informativo sobre a intervenção, uso das avaliações regulares da fidelidade como retorno às equipes, tipo de organização participante (serviços voluntários podem apresentar mais motivação), realização das mudanças estruturais necessárias para a intervenção rapidamente ${ }^{26,31,37}$. Já os fatores relacionados à baixa fidelidade foram: resistência dos profissionais em implementar o programa e em abandonar velhas práticas de tratamento, serviços em que as antigas práticas são conflitantes com a intervenção a ser implementada, restrição de financiamento e mau relacionamento com as autoridades em saúde no local da intervenção ${ }^{26,27,29,31}$. Tais fatores apontam para importantes aspectos que devem ser priorizados num processo de implementação, a fim de que não haja prejuízo em seus desfechos. Destaque para a gestão do grupo, pois se observou que um gerenciamento experiente e atuante poderia prevenir a alta rotatividade da equipe envolvida. Idealmente, todos os profissionais deveriam possuir experiência, mas tal atributo torna-se fundamental para a designação dos cargos de coordenação e supervisão. 0 treinamento de qualidade antes do início da intervenção, com total esclarecimento sobre os procedimentos a serem realizados, também pode ser útil para que não haja resistência ou retorno às práticas anteriores pela equipe. Portanto, dar maior atenção a alguns simples detalhes no processo da implementação pode implicar positivamente na fidelidade à intervenção.

\section{CONCLUSÃO}

Por meio da revisão realizada, pôde ser percebido que mais do que simplesmente documentar o quanto do modelo original foi seguido, a avaliação da fidelidade também fornece informações relativas à população-alvo, aos aspectos culturais do local da intervenção e aos desfechos esperados, o que permite a realização de adequações necessárias durante o seu curso, a fim de que os procedimentos não se distanciem do protocolo e seja alcançada excelência no processo de implementação.

Mais estudos serão necessários para avaliar o quanto os fatores facilitadores e as barreiras da implementação influenciam na sustentabilidade das intervenções ao longo do tempo, que estratégias podem superar as dificuldades observadas, que componentes do modelo são indispensáveis e se diferentes modelos de implementação são úteis para diferentes tipos de organizações.

A avaliação de intervenções psicossociais com o devido rigor científico não permite a descaracterização do modelo, 
pois, desse modo, não seria possível afirmar ao final do tratamento que a mesma intervenção foi igualmente oferecida a todos os participantes. Isso impossibilitaria uma análise fidedigna de sua efetividade. Embora as particularidades de cada população sejam extremamente importantes na composição de uma intervenção ideal, é crucial que, primeiramente, determinado modelo possa ser seguido de modo fidedigno. Então, a partir desses seus resultados, as adaptações poderiam ser racionalmente direcionadas para os diferentes contextos populacionais.

Neste estudo, foram consultadas importantes bases de dados. Porém, em se tratando de uma revisão global, o fato de não terem sido consultadas todas as bases de dados existentes pode ser citado como uma limitação do estudo.

\section{CONTRIBUIÇÕES INDIVIDUAIS}

A autora atuou na concepção e elaboração do artigo, na análise e interpretação dos dados e na revisão crítica do conteúdo intelectual e aprovou sua versão final a ser publicada.

\section{CONFLITOS DE INTERESSE}

A autora declara não haver conflitos de interesse.

\section{AGRADECIMENTO}

À médica-residente Louise Verdolin, pelo auxílio na organização e revisão da bibliografia pesquisada.

\section{REFERÊNCIAS}

1. World Health Organization. The global burden of disease: 2004 update. Geneva: World Health Organization; 2008.

2. Kohn R, Saxena S, Levav I, Saraceno B. The treatment gap in mental health care. Bull WHO. 2004;82(11):858-66.

3. Chowdur R, Dharitri R, Kalyanasundaram S, Suryanarayana R. Efficacy of psychosocial rehabilitation program: The RFS experience. Indian J Psychiatry. 2011;53(1):45-8.

4. McHugo G, Drake R, Whitley R, Bond G, Campbell K, Rapp C, et al. Fidelity outcomes in the National Evidence-Based Practices Project. Psychiatr Serv. 2007;58(10):1279-84.

5. Bond G, Evans L, Salyers M, Williams J, Kim H. Measurement of fidelity in psychiatric rehabilitation. Ment Health Serv Res. 2000;2(2):75-87.

6. Waltz J, Addis M, Koerner K, Jacobson N. Testing the integrity of a psychotherapy protocol: assessment of adherence and competence. J Consult Clin Psychol. 1993;61(4):620-30.

7. Proctor E, Silmere H, Raghavan R, Hovmand P, Aarons G, Bunger A, et al. Outcomes for implementation research: conceptual distinctions, measurement challenges and research agenda. Adm Policy Ment Health. 2011;38(2):65-76.

8. Teague G, Bond G, Drake R. Program fidelity in assertive community treatment: development and use of a measure. Am J Orthopsy. 1998;68(2):216-32.

9. Monroe-DeVita M, Teague G, Moser L. The TMACT: a new tool for measuring fidelity to assertive community treatment. J Am Psychiatr Nurses Assoc. 2011;17(1):17-29.
10. McHugo G, Drake R, Teague G, Xie H. Fidelity to assertive community treatment and client outcomes in the New Hampshire dual disorders study. Psychiatr Serv. 1999;50(6):818-24.

11. McHugo G, Hargreaves W, Drake R, Clark R, Xie H, Bond G, et al. Methodological issues in assertive community treatment studies. Am J Orthopsy. 1998;68(2):246-60.

12. Perepletchikova F, Treat T, Kazdin A. Treatment integrity in psychotherapy research: analysis of the studies and examination of the associated factors. J Consul Clin Psychol. 2007;75(6):829-41.

13. Howard L, Oram S, Galley H, Trevillion K, Feder G. Domestic violence and perinatal mental disorders: a systematic review and meta-analysis. PLoS Med. 2013;10(5):1452-68.

14. Ablon S, Jones E. Psychotherapy process in the National Institute of Mental Health Treatment of Depression Collaborative Research Program. J Consult Clin Psychol. 1999;67(1):64-75.

15. Carroll K, Nich C, Sifry R, Nuro K, Frankforter T, Ball S, et al. A general system for evaluating therapist adherence and competence in psychotherapy research in the addictions. Drug Alcohol Depend. 2000;57(3):225-38.

16. Macias C, Propst R, Rodican C, Boyd J. Strategic planning for ICCD clubhouse implementation: development of the Clubhouse Research and Evaluation Screening Survey (CRESS). International Center for Clubhouse Development. Ment Health Serv Res. 2001;(3)3:155-67.

17. Salyers M, Bond G, Teague G, Cox JF, Smith ME, Hicks ML, et al. Is it ACT yet? Real-world examples of evaluating the degree of implementation for Assertive community treatment. J Behav Health Serv Res. 2003;30(3):304-20.

18. Hayes RA, Andrews N, Baron-Jeffrey M, Conley C, Gridley K, Norman R, et al. Service enhancement to a dual-diagnosis population: mental illness/substance abuse (MISA). Qual Manag Health Care. 2003;12:133-50.

19. Fiander M, Burns T, McHugo G, Drake R. Assertive community treatment across the AtIantic: comparison of model fidelity in the UK and USA. Br J Psychiatry. 2003;182:248-54.

20. Oshima I, Cho N, Takahashi K. Effective components of a nationwide case management program in Japan for individuals with severe mental illness. Community Ment Health J. 2004;40(6):525-37.

21. McGrew J, Griss M. Concurrent and predictive validity of two scales to assess the fidelity of implementation of supported employment. Psychiatr Rehabil J. 2005;29(1):41-7.

22. Falloon I, Economou M, Palli A, Malm U, Mizuno M, Murakami M; Optimal Treatment Project Collaborative Group. The clinical strategies implementation scale to measure implementation of treatment in mental health services. Psychiatr Serv. 2005;56(12):1584-90.

23. Essock S, Covell N, Shear K, Donahue S, Felton C. Use of clients' self-reports to monitor project liberty clinicians' fidelity to a cognitive-behavioral intervention. Psychiatr Serv. 2006;57(9):1320-3

24. Milby J, Schumacher J, Freedman M, Lester K, Netson K, Norwood W, et al. A progressive process for technology transfer of a complex, effective psychosocial intervention. Addict Disord Their Treat. 2007;6:187-96.

25. Van Voorhees B, Ellis J, Gollan J, Bell C, Stuart S, Fogel J, et al. Development and process evaluation of primary care internet-based intervention to prevent depression in emerging adults. Prim Care Companion J Clin Psychiatry. 2007;9(5):346-55.

26. Bond G, McHugo G, Becker D, Rapp C, Whitley R. Fidelity of supported employment: lessons learned from the National Evidence- Based Practice Project. Psychiatr Rehabil J. 2008;31(4):300-5.

27. Brunette M, Asher D, Whitley R, Lutz W, Wieder B, Jones A, et al. Implementation of integrated dual disorders treatment: a qualitative analysis of facilitators and barriers. Psychiatr Serv. 2008;59(9):989-95.

28. Randall C, Biggs B. Enhancing therapeutic gains: examination of fidelity to the model for the intensive mental health program. J Child Fam Stud. 2008;17:191-205.

29. Boyce M, Seeker J. Factors influencing the delivery of evidence-based supported employment in England. Psychiatr Rehabil J. 2008;31(4):360-6.

30. Forsner T, Wistedt A, Brommels M, Forsell Y. An approach to measure compliance to clinical guidelines in psychiatric care. BMC Psychiatry. 2008;8:64.

31. Woltmann E, Whitley $R$, Mchugo $G$, Brunett M, Torrey $W$, Coots $L$, et al. The role of staff turnover in the implementation of evidence-based practices in mental health care. Psychiatr Serv. 2008;59(7):732-7. 
32. Catty J, Lissouba P, White S, Becker T, Drake RE, Fioritti A, et al. Predictors of employment for people with severe mental illness: results of an international six-centre randomised controlled trial Br J Psychiatry. 2008;192(3):224-31.

33. Martino S, Ball S, Nich C, Frankforter T, Carroll K. Community program therapist adherence and competence in motivational enhancement therapy. Drug Alcohol Depend. 2008;96(1-2):37-48.

34. Wilson D, Crisanti A. Psychometric properties of the dual-disorder treatment fidelity scale: inter-rater reliability and concurrent validity. Community Ment Health J. 2009:45:171-8.

35. Matejkowski J, Draine J. Investigating the impact of housing first on ACT fidelity. Community Ment Health J. 2009:45(3):6-11.

36. Killaspy H, Johnson S, Pierce B, Bebbington P, Pilling S, Nolan F, et al. Successful engagement: a mixed methods study of the approaches of assertive community treatment and community mental health teams in the REACT trial. Soc Psychiatry Psychiatr Epidemiol. 2009;44(7):532-40.
37. Long M, Grubaugh A, Elhai J, Cusak K, Knapp R, Frueh B. Therapist fidelity with an exposure-based treatment of PTSD in adults with schizophrenia or schizoaffective disorder. J Clin Psychol. 2010;66(4):383-93.

38. McGrew J, Stull L, Rollins A, Salyers M, Hicks L. A comparison of phone-based and onsite assessment of fidelity for assertive community treatment in Indiana. Psychiatr Serv. 2011;62(6):670-4.

39. Aarons G, Fettes D, Sommerfeld D, Palinkas L. Mixed methods for implementation research: application to evidence-based practice implementation and staff turnover in community-based organizations providing child welfare services. Child Maltreat. 2012;17(1):67-79.

40. Randall $G$, Wakefield $P$, Richards D. Fidelity to assertive community treatment program standards: a regional survey of adherence to standards. Community Ment Health J. 2012:48(2):138-49. 